\title{
Plazebo: Ärgernis oder gleichermassen wichtige Rückseite der Medaille?
}

Wenn überzeugte Naturheilkundler oder Komplementärmediziner mit eingefleischten Schulmedizinern ins Diskutieren kommen, dann prallen konzeptionelle Welten aufeinander. Reduktionisten schimpfen die einen, die für sich selbst eine ganzheitliche (holistische) Sicht der Dinge in Anspruch nehmen - Plazebomediziner lästern die anderen, die für sich eine «objektive, wissenschaftliche» Sicht der Dinge reklamieren. Plazebo - ein Reizwort, ein Schimpfwort. Synonym für Scharlatanerie, Quacksalberei, Kurieren mit geschlossenen Augen, Betrug am Patienten? Oder einfach inhärent unethisch, da im Wesentlichen ein Phantom, ein Mythos - gegen dessen angebliche «Power» [1] die «Evidence» aus vielen Studien spricht [2]?

In der Vergangenheit mit Abstand am häufigsten eingesetzt wurden Plazebos in der Arzneimittelforschung, da die $\mathrm{Zu}-$ lassungsbehörden der meisten Industrieländer grundsätzlich mindestens zwei randomisiert kontrollierte Studien fordern, bei denen in der Kontrollgruppe ein Medikament ohne einen arzneilich wirksamen Stoff einem äusserlich identischen Medikament gegenübergestellt wird, das als einzigen Unterschied den Wirkstoff enthält, für den die Zulassung beantragt wird.

Es geht dabei ausschliesslich um die Frage: Was bewirkt dieser Wirkstoff? Deshalb ist es auch logisch, konsequent und richtig, dass alle anderen potenziellen Einflussfaktoren ausgeschaltet werden müssen, vor allem die Erwartungshaltung seitens des Patienten und eventuelle, auch un- oder unterbewusste suggestive Einflüsse seitens des Behandlers. Das wird erreicht durch die sog. doppelte Verblindung, deren Ziel es ist, zu «maskieren», welcher Patient welches der beiden Präparate, «Verum» oder «Plazebo», erhält. Ein ausgeprägter Plazeboeffekt ist bei Arzneimittelstudien verständlicherweise nichts weniger als erwünscht, denn sein Anteil am Gesamteffekt geht zu Lasten des spezifischen Effektes. Auch damit mag das eher schlechte Image des Plazeboeffektes zusammen hängen. Gut möglich auch, dass die Ergebnisse des systematischen Reviews von Hrobjartsson und Gotzsche [2] («we found little evidence in general that placebos had powerful clinical effects») nicht nur durch einen relevanten Publication Bias, sondern auch durch den vorherrschenden Kontext Arzneimittelstudie verzerrt sind. Wenig Klarheit scheint auch konzeptionell zu bestehen, wir selbst fanden z.B. in einer systematischen MedlineAnalyse Mitte der 90er Jahre, dass in 40\% der Publikationen, in denen die beiden Begriffe «Plazebo» und «unbehandelt» verwendet wurden, diese synonym verwendet wurden [3]. Dabei gibt es bemerkenswerte experimentelle Hinweise, dass nicht nur der Wirkstoff einen relevanten Effekt verursachen kann, sondern dass dies auch «unspezifische Wirkfaktoren» können. Ein Plazebo zum Einreiben wirkt besser als eines zum Einnehmen in Tropfenform [4]. Fast jeder hat die klinische Erfahrung gemacht, dass eine Injektion ohne Wirkstoff eine stärkere Wirkung hat als die perorale Form - und in der Lage ist, (zumindest temporär) klinische relevante Effekte zu induzieren. Selbst das Immunsystem kann durch bestimmte Reize nachweislich konditioniert werden [5].

Der Anteil des unspezifischen Effektes am Gesamteffekt kann auch dort erheblich sein, wo man den spezifischen Effekt klar als den entscheidenden erwartet: bei invasiven Massnahmen. Schon Ende der 50er Jahre wurde in einer bemerkenswerten Studie gezeigt, dass bei Patienten mit koronarer Herzerkrankung die chirurgische Unterbindung der A. mammaria interna, die nach damaliger Vorstellung im Sinne eines «steal»-Phänomens Anfälle von Angina pectoris verursachen oder verschlimmern sollte, zu einer ebenso ausgeprägten Verbesserung des klinischen Bildes führte wie ein kleiner Hautschnitt alleine [6]. In einer anderen, jüngst ebenfalls im «New England Journal of Medicine» publizierten Studie mit $180 \mathrm{~Pa}$ tienten mit Kniegelenksarthrose unterschieden sich die Ergebnisse in Bezug auf Schmerz und Funktion im Verlauf von zwei Jahren nach einer Arthroskopie ebenfalls nicht vonein-

\begin{tabular}{ll}
\hline KARGER & @ 2004 S. Karger GmbH, Freiburg \\
$\begin{array}{l}\text { Fax +497614520714 } \\
\begin{array}{l}\text { E-mail Information@Karger.de } \\
\text { www.karger.com }\end{array}\end{array}$ & $\begin{array}{l}\text { Accessible online at: } \\
\text { www.karger.com/fkm }\end{array}$
\end{tabular}

Prof. Dr. med. Karl-Ludwig Resch

Forschungsinstitut für Balneologie und Kurortwissenschaft Bad Elster Lindenstr. 5

D-08645 Bad Elster

Tel. +49 37437-55750, Fax -3185

E-mail K.L.Resch@t-online.de 
ander, gleichgültig, ob ein Débridement oder eine Lavage des Kniegelenkes durchgeführt wurde, oder ein Débridement nur simuliert und lediglich drei kleine Hautinzisionen gemacht wurden [7]. In einer niederländischen Studie, bei der bei einem Teil der Patienten mit chronischen Bauchschmerzen im Rahmen einer Laparoskopie eine Adhäsiolyse durchgeführt, beim anderen Teil nichts gemacht wurde, gaben die Patienten beider Gruppen ohne Unterschied eine substanzielle Besserung ihrer Schmerzen und eine signifikante Verbesserung der Lebensqualität an [8].

Mitte Mai 2004 wurden in Berlin erste Ergebnisse von zwei der drei Modellvorhaben vorgestellt, die für die Krankenkassen klären sollen, ob ein komplementärmedizinisches «invasives» Verfahren, die Akupunktur, bei bestimmten Erkrankungen wirksam, sicher und wirtschaftlich ist (der Bundesausschuss Ärzte/Krankenkassen hatte vor einigen Jahren mit dem Hinweis auf eine unzureichende Evidenzlage die Akupunktur von der Liste der erstattungsfähigen Therapien gestrichen). Es soll in diesem Zusammenhang nicht näher darauf eingegangen werden, dass es sich um die mit Abstand weltweit grösste Studie zur Akupunktur handelt, an der innerhalb von zwei Jahren etwa 3\% aller gesetzlich Krankenversicherten teilgenommen haben. Es soll auch nicht darauf eingegangen werden, dass die Ergebnisse teilweise recht eindrucksvoll und deutlich sind (vor allem für Akupunkturbehandlung der Kniegelenksarthrose). Interessant ist in diesem Zusammenhang vielmehr ein Aspekt, der den beteiligten Wissenschaftlern wie den an der Veranstaltung Anwesenden offensichtlich gleichermassen viel Kopfzerbrechen macht. Bei zwei von drei Indikationsbereichen (chronischer Rückenschmerz und Migräne) führte die Akupunktur zwar zu einer erheblichen Verbesserung der Symptomatik im Vergleich zu einer unbehandelten Gruppe (die auf die Behandlung wartete), das Ausmass der Verbesserung unterschied sich aber nur marginal von dem einer dritten Gruppe, die eine «Minimalakupunktur» erhielt. So kreiste denn auch ein Gutteil der Diskussion um das leidige (um nicht zu sagen, ärgerliche) Phänomen Plazebo, rangen Beteiligte und akupunktierende Ärzte - Ungemach vor Augen - um exkulpierende Erklärungen.

Während sich bei den oben vorgestellten aktuellen chirurgischen Studien tatsächlich die Frage stellt, ob vor dem Hintergrund der Studienergebnisse eine so grosszügige Indikationsstellung für Arthroskopie bzw. Laparoskopie künftig noch zu rechtfertigen ist, scheint mir die vorliegende Ergebniskonstellation zur Akupunktur in eine ganz andere Richtung zu deuten. Sie ist z.B. durchaus geeignet, die Grundthese zu bestätigen, dass es sich bei der Akupunktur um eine Reiz-Reaktions-Therapie handelt. Das Konzept «Reiz-Reaktion» bzw. «Reiz-Regulation» ist das Fundament, das prägende Wesensmerkmal der meisten empirisch gewachsenen, ethnisch-kulturell verankerten Medizinsysteme von der Klassischen Naturheilkunde in Europa über das indische Ayurveda bis zur Traditionellen Chinesischen Medizin (und damit auch der Akupunktur), um nur die populärsten zu nennen. Schon die römischen Ärzte Celsus (30 v.Chr. - 38 n.Chr.) und Galen (130-201 n.Chr.) beschrieben etwa die begrenzte Anzahl von (recht unspezifischen) Antwortmöglichkeiten des Körpers auf inflammatorische Reize, nämlich Rubor, Calor, Tumor, Dolor und Functio laesa. Auch die Reaktion unseres vegetativen Nervensystems auf ganz unterschiedliche Stimuli ist immer entweder Parasympathikus- oder Sympathikus-dominiert.

In einem 2003 in der «Forschenden Komplementärmedizin und Klassischen Naturheilkunde» erschienenen Editorial wurde bereits darauf hingewiesen, dass gerade im Reiz-Reaktions-Geschehen eine klare Abgrenzung zwischen den Konstrukten «spezifisch» und «unspezifisch» schwierig ist bzw. beide häufig parallel wirksam werden [9]. Vor wenigen Wochen wurde nun in der Zeitschrift «Science» eine Studie publiziert, die für das Verständnis dieses Zusammenspiels möglicherweise bahnbrechende neue Erkenntnisse liefert [10]. Die Entwicklung der Kernspintomographie erlaubt mittlerweile funktionelle Aufnahmen auch im Bereich des Zentralnervensystems. Damit lassen sich z.B. Gehirnaktivitäten exakt lokalisieren und quantifizieren. In einer der Studien wurden Probanden, denen erklärt wurde, sie nähmen an der Testung einer neuen, analgetisch wirksamen Salbe teil, in einem komplexen Studienansatz wiederholt schmerzhaften elektrischen Stimuli ausgesetzt. Verglichen wurden die Gehirnaktivitäten in definierten Arealen bei schmerzhaften Reizen a) ohne Salbe, b) mit Salbe und der Information, es handele sich um eine wirkungslose Salbe zur Kontrolle und c) mit Salbe und der Information, es handele sich um die zu testende, analgetisch wirksame Salbe (die tatsächlich keinerlei analgetische Wirkung hatte). Im Ansatz c) wurde eine erheblich niedrigere Aktivität in den «schmerzsensitiven» Gehirnregionen beobachtet, gleichbedeutend mit einem erniedrigten wahrgenommenen Schmerzniveau. Ausserdem konnte gezeigt werden, dass die Erwartung einer analgetischen Wirkung die Aktivität in den schmerzverarbeitenden Regionen des Gehirns (insbesondere des präfrontalen Cortex) erhöht. Je höher diese Aktivität, umso niedriger die Aktivität in den schmerzsensitiven Arealen während des Stimulus, oder, wie in einer begleitenden $\mathrm{Zu}$ sammenfassung formuliert, «anticipation of pain relief is intimately tied with actual pain reduction».

Von einem technischen Instrument, etwa einem Fieberthermometer, ist die reproduzierbare, präzise und valide (geeichte) Wiedergabe einer definierbaren, vorgegebenen externen physikalischen Eigenschaft zu fordern - vergleichbar dem Konstrukt des spezifischen Effekts. Unsere Wahrnehmung unterscheidet sich davon offensichtlich fundamental [z.B. 11], und damit ist die Erwartung eines Patienten in vielen Bereichen eben nicht durch eine Intervention mit gut dokumentierter Wirksamkeit ausschliesslich im Sinne eines spezifischen Effektes hinreichend zu bedienen. Dies wird unterstrichen durch die offensichtlich massive Nachfrage (sprich: Druck) nach Akupunktur seitens der Patienten, die für mich wohl die entscheidende Motivation darstellte, dass nach der «negativen» Entscheidung des Bundesausschusses eine derart umfassende 
Evaluation der Akupunktur in die Wege geleitet und finanziert wurde.

Auf der Ebene der Versorgung scheint mir deshalb die Fokussierung auf die Ebene des spezifischen Effektes, also auf die (teilweise wenig ausgeprägten) Unterschiede zwischen Verum- und Minimalakupunktur, inadäquat und von allenfalls sekundärer Bedeutung. Überträgt man die Ergebnisse der Studie von Wager et al. [10] auf die Akupunktur, würde das bedeuten, dass der insgesamt erzielbare Gesamteffekt ohne die «unspezifische» Komponente der Akupunktur auch nicht annähernd erreicht werden kann, umgekehrt aber dieser auch nicht ohne die Akupunktur erreicht wird. Das bedeutet auch, unspezifisch heisst nicht beliebig oder auswechselbar, es bedarf vielmehr u.U. sehr spezifischer Massnahmen, um gezielt auch das «unspezifische» Potenzial anzusprechen und zu mobilisieren.

Letztere Frage eröffnet eine hochspannende, bislang kaum gezielt untersuchte Dimension [12]. Welche spezifischen Kenntnisse sind erforderlich, um einen klinisch akzeptablen Effekt antizipierbar und reproduzierbar zu erzielen? Dies scheint mir aber eher eine Frage für die Grundlagenforschung zu sein als für die Versorgung, da es im Wesentlichen um eine technische Fragestellung, nämlich die Optimierung des spezifischen Effektes geht. Und da wäre nicht nur der Stand der Akupunkteure, genauer gesagt der Akupunkturschulen aufgerufen die gleiche Frage ist für weite Bereiche der klinischen Medizin offen, für die Psychotherapie wie die Massage, für einen Gutteil der Pharmakotherapie ebenso wie für invasive Massnahmen (siehe oben).

Auf lange Sicht könnte dann der von vielen naturheilkundlichen bzw. komplementärmedizinischen, aber auch alterna- tivmedizinischen Therapeuten reklamierte Anspruch der Ganzheitlichkeit, des «holistischen» Ansatzes, besser fassbar werden, könnte Komplexes, aber Seriöses leichter, zuverlässiger und besser von windiger Quacksalberei differenziert werden.

K.L. Resch, Bad Elster

\section{Literatur}

1 Ernst E, Saradeth T, Resch KL: The powerful placebo. Lancet 1991;337:611.

2 Hrobjartsson A, Gotzsche PC: Is the placebo powerless? An analysis of clinical trials comparing placebo with no treatment. N Engl J Med 2001;344:1594-1602.

3 Ernst E, Resch KL: The importance of placebo effects. JAMA 1995;273:283.

4 Saradeth T, Resch KL, Ernst E: Placebo treatment for varicosity: Don't eat it rub it. Phlebology 1994;9:63-66.

5 Goebel MU, Trebst AE, Steiner J, Xie YF, Exton MS, Frede S, Canbay AE, Michel MC, Heemann U, Schedlowski M: Behavioral conditioning of immunosuppression is possible in humans. FASEB J 2002;16:1869-1873.

6 Cobb LA, Thomas G, Dillard DH, Merendino KA, Bruce RA: An evaluation of internal-mammary artery ligation by a double-blind technique. N Engl J Med 1959;260:1115-1118.

7 Moseley JB, O'Malley K, Petersen NJ, Menke TJ, Brody BA, Kuykendall DH, Hollingsworth JC, Ashton CM, Wray NP: A controlled trial of arthroscopic surgery for osteoarthritis of the knee. N Engl J Med 2002;347:81-88.

8 Swank DJ, Swank-Bordewijk SCG, Hop WCJ, van Erp WFM, Janssen IMC, Bonjer HJ, Jeekel J: Laparoscopic adhesiolysis in patients with chronic abdominal pain: A blinded randomised controlled multi-centre trial. Lancet 2003;361:12471251

9 Gutenbrunner C: Unspezifische Stimuli - ein klassisches Missverständnis. Forsch Komplementärmed Klass Naturheilkd 2003;10:296-297.

10 Wager TD, Rilling JK, Smith EE, Sokolik A, Casey KL, Davidson RJ, Kosslyn SM, Rose RM, Cohen JD: Placebo-induced changes in fMRI in the anticipation and experience of pain. Science 2004;303:1162-1167 (zusätzliche Details: http:// www.sciencemag.org/cgi/content/full/303/5661/1162/DC1).

11 Saradeth T, Resch KL, Ernst E: Placebo-Effekte bei chronisch venöser Insuffizienz - Eine Meta-Analyse. Perfusion 1995;8:134-142.

12 Ernst E, Resch KL: Concept of true and perceived placebo effects. BMJ 1995; 311:551-553. 